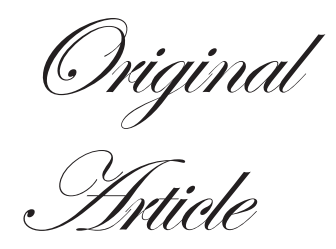

\title{
Prevalence of extra-esophageal cancers in patients with Barrett's esophagus and esophageal adenocarcinoma
}

\author{
Anjan Talukdar, ${ }^{1}$ Amitabh Chak, ${ }^{2}$ Tommy Lee, ${ }^{1}$ Xiang Fang, ${ }^{3}$ Patrice \\ Watson, ${ }^{4}$ Henry Lynch, ${ }^{4}$ Sumeet Mittal, ${ }^{1}$
}

\section{ABSTRACT}

Department of Surgery, ${ }^{1}$

Department of Statistics, ${ }^{3}$

Hereditary Cancer Center, ${ }^{4}$

Creighton University

School of Medicine,

Omaha, NE 68131;

Division of Gastroenterology and

Liver Disease, ${ }^{2}$

Case Western Reserve University, Cleveland, OH 44106,

USA.

Correspondence:

Dr. Sumeet Mittal

Email: $\underline{\text { skmittal@creighton.edu }}$
Background and Aim: To compare the prevalence of extra-esophageal cancers in patients diagnosed with Barrett's esophagus (BE) and esophageal adenocarcinoma (EAC) with SEER database.

Methods: Patients with BE and EAC are part of a NIH supported Familial Barrett's investigation involving personal and family history and pathology correlation recorded in the database. Data pertaining to extra-esophageal cancers in the proband was extracted into an excel datasheet for analysis. Expected prevalence obtained from SEER (Surveillance, Epidemiology and End Results) NIH database (1973-2006) for the general population, matched for age, was compared with our cohort. Chi-square test was used for statistical analysis.

Results: There were 1091 probands in the database of whom 876 had complete personal history. The mean age was 57.6 (5-84 years) with 807 Caucasians and 710 males. Overall incidence of extra-esophageal cancers was higher in our cohort when compared with the general population.

Conclusion: There is a strong association of certain cancer types in patients with $\mathrm{BE}$ and EAC. However, further epidemiologic and genetic research is needed for investigation and development of genetic fingerprints

KEYWORDS: Barrett's esophagus, esophageal adenocarcinoma, extra-esophageal cancer

\section{Introduction}

Esophageal adenocarcinoma (EAC) has an estimated prevalence of 7.7 per 100,000 in the US. ${ }^{1}$ The 5 -year survival after diagnosis is low ranging between 3 to $37 \%$ depending on disease stage. ${ }^{1}$ Risk factors for EAC have been described but association of extra-esophageal cancers in people diagnosed with EAC and Barrett's esophagus (BE) has not been studied extensively except for associations with colo-rectal cancer. ${ }^{2-6}$
Indentified risk factors include $\mathrm{BE}$, gastro-esophageal reflux disease, familial predisposition, ${ }^{7-8}$ obesity, ${ }^{9-11}$ smoking ${ }^{12}$ and alcohol use. ${ }^{12}$ The purpose of our study is to compare the prevalence of extra-esophageal cancers in patients diagnosed with BE and EAC with the SEER (Surveillance, Epidemiology and End Results) database. The SEER program is the authoritative source of information for various cancers. This 
may facilitate diagnosis of other cancers in patients diagnosed with $\mathrm{EAC}$ and $\mathrm{BE}$ which in turn may change management strategies.

\section{Methods}

Patients with BE and EAC are part of a NIH (National Institute of Health) supported Familial Barrett's RO1 investigation involving multiple centers throughout the USA including Case Western Reserve University, University of Pennsylvania, Creighton University, Mayo Clinic and the UNC.

Detailed personal, medical and family history and pathology investigations are recorded in the database. Patients with incomplete data were excluded. Data pertaining to extraesophageal cancers in the proband was extracted into an excel datasheet for analysis. Data pertaining extra-esophageal cancer was obtained by interviewing patients during clinic visits.
Expected prevalence obtained from SEER (Surveillance, Epidemiology and End Results) NIH database (1973-2006) for the general population, matched for age and sex, was compared with our cohort. The SEER registry is the authoritative source of information on cancer prevalence and survival in the United States. This program currently collects and publishes cancer prevalence and survival data from population-based cancer registries covering approximately 28 percent of the US population. ${ }^{1}$

Fisher's exact test was used for comparison of observed and expected frequencies ${ }^{13}$ when individual values were less than 5, otherwise Chi-square test was used. Paired T-test was used for comparison of age at the time of diagnosis of BE/EAC and other cancers. Age had normal distribution which was evaluated using SPSS (Statistical Package for the Social Sciences) statistical software, version 19.0. A p value $<0.05$ was considered significant.
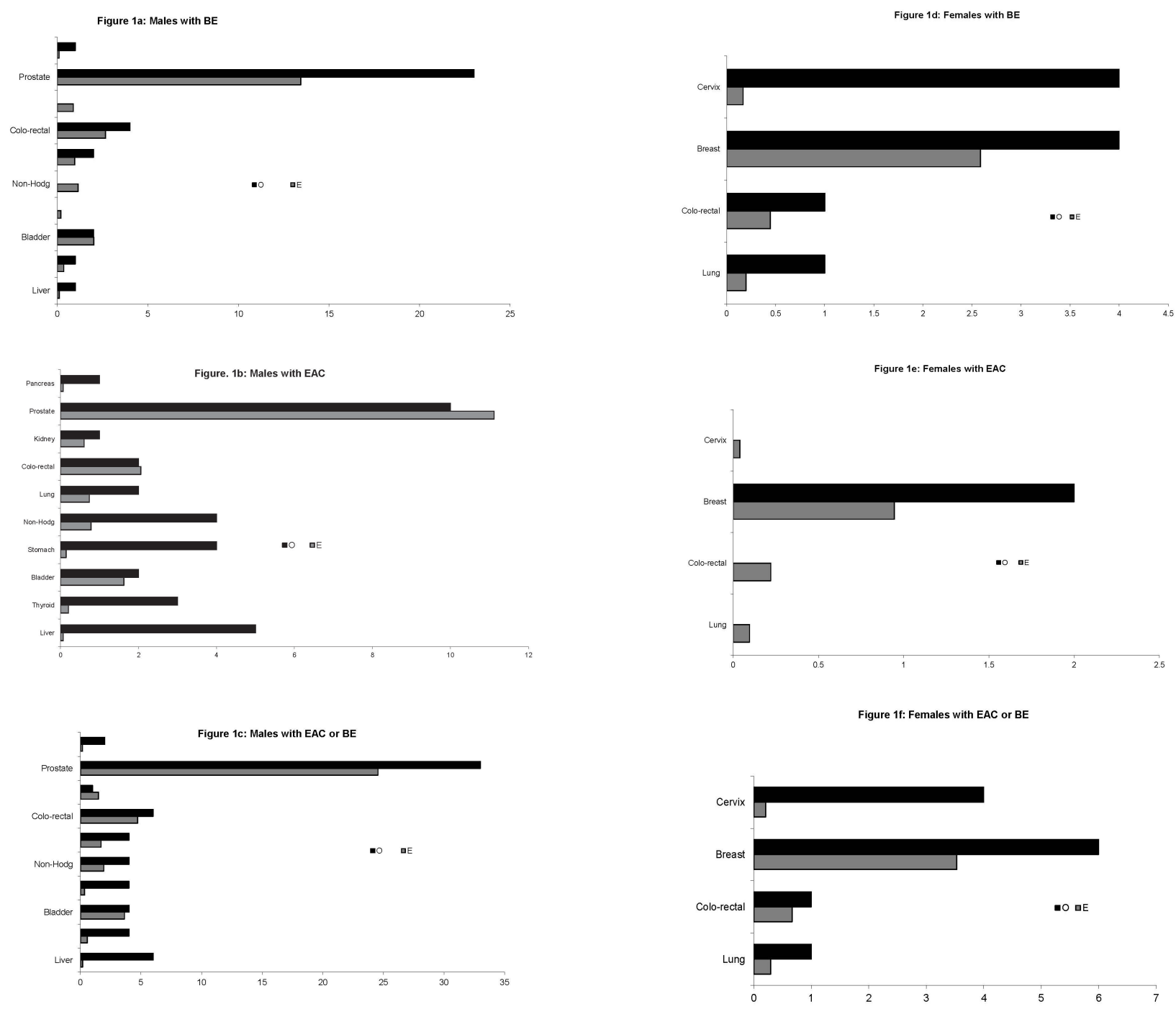

Figure1: Frequencies of extra-esophageal cancers (X-axis are absolute numbers) 


\section{Results}

There were 1091 probands in the database of whom $876(80 \%)$ had complete personal history. The median age \pm SD was $58 \pm$ 14 (5-84 years) with 807 Caucasians and 710 males. Subjects in $\mathrm{BE}$ and EAC groups were exclusive of each other in our study. We did not have data about the number of patients in the EAC group who had prior history of Barrett's. Overall prevalence of extra-esophageal cancers was higher in our cohort when compared with the population from the SEER database (9.9\% vs. $5.5 \%, \mathrm{p}=0.0000223$ ). Males with $\mathrm{EAC}$ and $\mathrm{BE}$ had a statistically higher prevalence of extra-esophageal cancers (observed vs. expected: $10.4 \%$ vs. $6.0 \%, \mathrm{p}=0.0002$ ) when compared with the population from the SEER database.

Males diagnosed with BE (Figure 1a) had a higher prevalence of prostate cancer $(\mathrm{p}=0.0621)$. Males with EAC (Figure 1b) had higher prevalence of liver cancer ( $p=0.0306$ ), and cancer of the stomach $(\mathrm{p}=0.0617)$. Combined as a group, males with EAC and BE (Figure 1c), had a higher prevalence of liver cancer ( $\mathrm{p}=0.0154)$, and stomach cancer $(\mathrm{p}=0.0622)$.

Females diagnosed with BE (Figure 1d) had a higher prevalence of cervical cancer ( $\mathrm{p}=0.0609)$. Combined as a group, females with EAC and BE (Figure 1f), had a higher prevalence of cervical cancer $(\mathrm{p}=0.0612)$.

The age at diagnosis of EAC and extra-esophageal cancers in the same patients were $70 \pm 12$ and $65 \pm 12$ years (median \pm $\mathrm{SD})$, respectively ( $\mathrm{p}=0.0002, \mathrm{n}=35)$. The age at diagnosis of $\mathrm{BE}$ and extra-esophageal cancers in the same patients were $62 \pm 13$ and $61 \pm 15$ years (median $\pm S D)$, respectively $(\mathrm{p}=0.219, \mathrm{n}=52)$.

\section{Discussion}

Prevalence of extra-esophageal cancers was higher in our cohort of patients with $\mathrm{BE}$ and $\mathrm{EAC}$ when compared with the population from the SEER database. Males diagnosed with BE had a higher prevalence of prostate cancer. Males diagnosed with EAC had a higher prevalence of liver cancer and cancer of the stomach. Females diagnosed with BE had a higher prevalence of cervical cancer.

Interest in hereditary cancer has piqued the concern of oncologists, surgeons, and geneticists, as well as high-risk patients. This, in turn, has fostered greater interest in examining in detail a family's pedigree for evidence of syndrome-related cancers that may indicate the presence of a hereditary cancer syndrome. In some settings, however, the cancer family history may pose a challenge due to variations in gene penetrance and expressivity of the phenotype which may then pose potential diagnostic confounders and therein prove to be a challenge to hereditary cancer syndrome diagnosis. However, the gestalt of clinical, pathology, molecular genetics and genealogic information can be used to great advantage in what we refer to as "pattern recognition", based upon the extra-esophageal cancer types that may ultimately distinguish hereditary esophageal cancer syndromes.

Clustering of esophageal cancer with cancers of oral cavity, pharynx, lung, bronchus, kidney, renal pelvis, colon, rectum, pancreas and breast has been observed by multiple studies. ${ }^{13-}$

${ }^{17}$ Environmental risk factors such as alcohol, tobacco and dietary heterocyclic amines has been found to be significantly associated with esophageal cancer. ${ }^{13,17,18}$ All of the associated cancers cannot be explained with known environmental risk factors which brings in the possibility of some unknown factor/ s playing a role in clustering of these cancers. Genetic factors may be responsible in selected cases. The frequency of EAC has been seen to vary among various groups across the world. ${ }^{16} \mathrm{Up}$ regulation of CDX2 (caudal related homebox gene 2) and down regulation of PITX1 (pituitary homebox 1) expression by RT-PCR (reverse-transcriptase PCR) has been shown to be associated with Barrett's and Barrett's associated EAC. ${ }^{19}$ Familial clustering implicating a genetic role has also been seen. ${ }^{20,21}$ P62 expression and detection of autoantibody in the serum of patients with EAC, gastric cancer and large intestinal cancer along with hepatocellular carcinoma has also been documented. ${ }^{22}$ These observations may suggest the possibility of a pattern of clustering of certain cancers with EAC, which share common genetic characteristics with environmental influences resulting in the final phenotypic expression.

We have found similar clustering of certain cancers with EAC, with males and females showing different patterns, which may be due to hormonal influences. Females had increased association with cervical cancer. This needs further investigation, which is beyond the scope of our current study.

The limitations of our study include inability to obtain complete data on all the patients diagnosed with BE and EAC. These patients may have had other cancers, which might have influenced the results somewhat differently. While this is a retrospective study, all data were collected prospectively. Further prospective studies are needed to validate our preliminary results where we need to follow all our patients and their extended families. We will need to determine accurate temporal relationships of these extra-esophageal cancers with 
BE/EAC. Furthermore, we do not have gene mapping and environmental risks of these individuals, which would have resulted in a better understanding of the cancer-clustering pattern coupled with its potential etiologic and pathogenic significance. Another possible reason for the higher prevalence of extra-esophageal cancers is a diagnostic bias due to closer follow-up and surveillance of patients with Barrett's or EAC compared to the general population. ${ }^{6}$ We realize that the annual prevalence of these cancers are low and large population based study needs to be done for validation. Statistical significance was not achieved in stomach and cervical cancer due to the small numbers but we could appreciate a trend when compared to other cancers.

\section{References}

1. Altekruse SF, Kosary CL, Krapcho M, Neyman N, Aminou R, Waldron W, et al, editors. SEER Cancer Statistics Review, 19752007, National Cancer Institute. Bethesda, MD, http:// seer.cancer.gov/csr/1975_2007/, based on November 2009 SEER data submission, posted to the SEER web site, 2010.

2. Sontag SJ, Schnell TG, Chejfec G, O'Connell S, Stanley MM, Best W, et al. Barrett's oesophagus and colonic tumours. Lancet. 1985;1:946-9.

3. Howden CW, Hornung CA. A systematic review of the association between Barrett's esophagus and colon neoplasms. Am J Gastroenterol. 1995;90:1814-9.

4. Cauvin JM, Goldfain D, Le Rhun M, Robaszkiewicz M, Cadiot G, Carpentier S, et al. Multicentre prospective controlled study of Barrett's oesophagus and colorectal adenomas. Groupe d'Etude de l'Oesophage de Barrett. Lancet. 1995;346:1391-4.

5. Poorman JC, Lieberman DA, Ippoliti AF, Weber LJ, Weinstein WM.The prevalence of colonic neoplasia in patients with Barrett's esophagus: prospective assessment in patients 50-80 years old. Am J Gastroenterol. 1997;92:592-6.

6. de Jonge PJ, van Blankenstein M, Looman CW, Casparie MK, Meijer GA, Kuipers EJ. Risk of colorectal cancer in patients with Barrett's esophagus: A Dutch population-based study. Am J Gastroenterol. 2010;105:77-83.

7. Chak A, Faulx A, Kinnard M, Brock W, Willis J, Wiesner GL, et al. Identiûcation of Barrett's esophagus in relatives by endoscopic screening. Am J Gastroenterol. 2004;99:2107-14.

8. Romero Y, Cameron AJ, Schaid DJ, McDonnell SK, Burgart LJ, Hardtke CL, et al. Barrett's esophagus: prevalence in symptomatic relatives. Am J Gastroenterol. 2002;97:1127-32.
9. Chow WH, Blot WJ, Vaughan TL, et al. Body mass index and risk of adenocarcinomas of the esophagus and gastric cardia. $J$ Natl Cancer Inst. 1998;90:150-5.

10. Vaughan TL, Davis S, Kristal A, Thomas DB. Obesity, alcohol, and tobacco as risk factors for cancers of the esophagus and gastric cardia: adenocarcinoma versus squamous cell carcinoma. Cancer Epidemiol Biomarkers Prev. 1995;4:85-92.

11. Lagergren J, Bergstrom R, Nyren O. Association between body mass and adenocarcinoma of the esophagus and gastric cardia. Ann Intern Med. 1999;130:883-90.

12. Wu AH, Wan P, Bernstein L. A multiethnic population-based study of smoking, alcohol and body size and risk of adenocarcinoma of the stomach and esophagus (United States). Cancer Causes Control. 2001;12:721-32.

13. Das A, Thomas S, Zablotska LB, Neugut AI, Chak A. Association of esophageal adenocarcinoma with other subsequent primary cancers. J Clin Gastroenterol. 2006;40:405-11.

14. Ono K, Takenaka M, Yokoyama E, Oka S, Baba T, So T, et al. Clinical analysis of esophageal cancer associated with other primary cancers. Kyobu Geka. 2011;64:93-6; discussion 97-8.

15. Kumagai Y, Kawano T, Nakajima Y, Nagai K, Inoue H, Nara S,et al. Multiple primary cancers associated with esophageal carcinoma. Surg Today. 2001;31:872-6.

16. Shibuya H, Wakita T, Nakagawa T, Fukuda H, Yasumoto M. The relation between an esophageal cancer and associated cancers in adjacent organs. Cancer. 1995;76:101-5.

17. Rossini AR, Hashimoto CL, Iriya K, Zerbini C, Baba ER, MoraesFilho JP.Dietary habits, ethanol and tobacco consumption as predictive factors in the development of esophageal carcinoma in patients with head and neck neoplasms. Dis Esophagus. 2008;21:316-21.

18. Terry PD, Lagergren J, Wolk A, Steineck G, Nyrén O. Dietary intake of heterocyclic amines and cancers of the esophagus and gastric cardia. Cancer Epidemiol Biomarkers Prev. 2003;12:940-4.

19. Ghadirian P, Vobecky J, Vobecky JS. Factors associated with cancer of the oesophagus: an overview. Cancer Detect Prev. 1988;11:225-34.

20. Chattopadhyay I, Phukan R, Singh A, Vasudevan M, Purkayastha J, Hewitt S, et al. Molecular profiling to identify molecular mechanism in esophageal cancer with familial clustering. Oncol Rep. 2009;21:1135-46.

21. Chang-Claude J, Becher H, Blettner M, Qiu S, Yang G, Wahrendorf J. Familial aggregation of oesophageal cancer in a high prevalence area in China. Int J Epidemiol. 1997;26:1159-65.

22. Su Y, Qian H, Zhang J, Wang S, Shi P, Peng X. The diversity expression of p62 in digestive system cancers. Clin Immunol. 2005;116:118-23. 